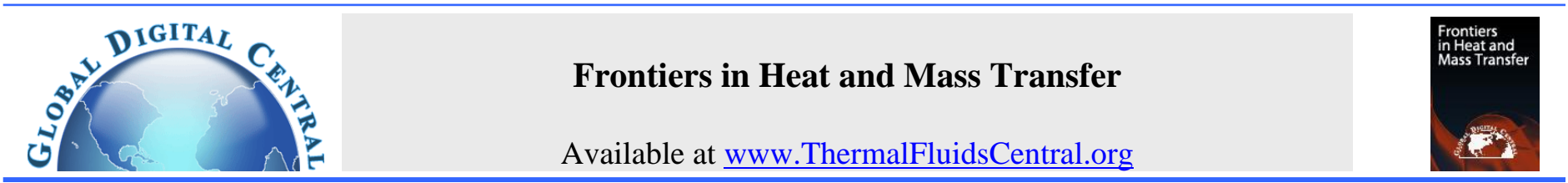

\title{
SIMULATIONS OF HYPERSONIC FLOW PAST A RE-ENTRY CAPSULE USING DSMC METHOD
}

\author{
R.V. Reji and S. Anil Lal ${ }^{\dagger}$ \\ Fluid Dynamics Lab, Department of Mechanical Engineering, College of Engineering Trivandrum, Kerala, India
}

\begin{abstract}
DSMC simulation of re-entry of an object with shape and size close to that of ISRO's Space Recovery Experiment (SRE) capsule has been analysed using the open-source tool dsmcFoam for three altitude conditions, viz. $85 \mathrm{~km}, 100 \mathrm{~km}$ and $115 \mathrm{~km}$, and for three angles of attack, viz. $0^{\circ}, 20^{\circ} \& 40^{\circ}$. The hypersonic free stream velocity of $8000 \mathrm{~m} / \mathrm{s}$ and boundary surface temperature of $300 \mathrm{~K}$ have been used for the simulations. The variation of parameters such as surface heat flux, surface pressure, shear stress, slip velocity, temperature jump and integrals such as total heat transfer, pressure drag and frictional drag are reported and discussed in this paper. The variation of temperature in the flow domain and along the stagnation line are able to clearly distinguish the shock structure and shock stand-off distance. The variation of local number density and the effects of such variations on the surface temperature and heat flux are discussed. A lower value for nose radius in SRE capsule is found to result in a higher value for peak heat flux compared to other re-entry capsules investigated.

Keywords: DSMC method, re-entry, hypersonic flow, rarefied flow.
\end{abstract}

\section{INTRODUCTION}

Shape is the most critical aspect to be considered for designing objects moving at hypersonic speed. The radius at the forward stagnation has a significant effect on the aero-thermal heating. The aero-thermal heating is inversely proportional to radius at the forward stagnation point. Hypersonic flow over objects are characterized by occurrence of a shock wave ahead of the body and large variations of density in the downstream region of the shock. The number density of a gas medium is the ratio of the total number of molecules to volume. Low value for the number density means rarefaction. Under rarefied condition, average distance travelled by gas molecules between two successive collisions called as mean free path, becomes large compared to the characteristic dimension of the object. Knudsen number $(\mathrm{Kn})$ is defined as the ratio of mean free path of the gas to characteristic dimension of the object, is commonly used to characterize the degree rarefaction in a flow. The continuum assumption and application of $\mathrm{N}-\mathrm{S}$ equation for fluid dynamic analysis are not strictly valid for high values of Knudsen number in the range $\mathrm{Kn}>0.1$. However continuum model using N-S equation in conjunction with slip boundary conditions has been extensively applied for flows up to $\mathrm{Kn}<0.2$ (Anderson, 2006).

For re-entry vehicles moving at hypersonic speed, rarefied regions of low number density can be observed in the flow domain. This situation arise mainly due to two reasons (a) lower value of free stream number density (b) displacement of the molecules away from some portions of the body surface in the downstream of bow shock. Under these situations, the value of free stream as well as some local values of $\mathrm{Kn}$ encountered in re- entry objects from higher altitudes may become greater than 0.2 and results in invalidity of continuum assumption and Navier-Stokes equations (An- derson, 2006; Bird, 1994). An equation valid for a wide range of values of $\mathrm{Kn}$ is the Boltzmann equation (Bird, 1994; Cercignani, 2000). Direct solution of Boltzmann equation involves computation of large number of molecules and hence impractical. A very simple and computationally affordable alternate method for solution of rarefied flows consists of random sampling of gas molecules. The method of solution is known as Direct Simulation Monte Carlo (DSMC). It is a statistical method which emulate the physical processes modeled in Boltzmann equation. The present work is concerned with analysis of hypersonic flow over a re-entry capsule.

The importance of the present work as an extension to the current state of knowledge is in relation to the state of the art Indian scenario. As a forerunner to this, a brief review of literature on development of DSMC method and simulations of high speed flow over objects are given here. Bird (1994) introduced the traditional algorithm and code for DSMC, that use a common cell based approach for collision calculation and macroscopic averaging. The open source versions of DSMC codes written in FORTRAN language are suitable for flow over regular geometries only. After introduction of DSMC method by Bird in 1962, a large number of studies have reported developments in DSMC method, algorithms and revealing the prediction of high speed flow using molecular models consisting of different levels of complexities. Chen and Boyd (1996) have investigated the statistical error in DSMC simulations using Bird's code and recommended minimum number of particles in a sampling cells in relation to the number of sampling steps. Bird (1994) recommends an optimum number of 8 particles in collision cells for better performance. These requirements for accurate simulations using DSMC method has led

${ }^{\dagger}$ Corresponding author. Email: anillal@cet.ac.in 
to the developments of a number of variants to the traditional methods. Bird [(Bird, 2007; Gallis et al., 2009) reported the sophisticated DSMC algorithm with new features for the purpose of improving the computational speed and reducing discretization error, which is not an open source software. Another important development focusing on improving the probabilistic computation of collision is due to LeBeau et al. (2003). They introduced a virtual-subcell method (VSC) for the selection of collision partners. In this method, the first particle in a collision cell is selected at random and the distances to all the remaining particles in the collision cell are calculated. Then, the nearest particle is selected as the partner for collision. A noted open source software development for DSMC using high potential utilities of open CFD software OpenFOAM is due to Scanlon et al. (2010). They developed a solver named as dsmcFoam. In this code an automatic sub-cell generation is applied for efficient calculation of nearest neighbour collisions. The dsmcFoam is available as an open source through GNU general public license. Though a large number of simulations using DSMC method can be found in literature, the simulation of high speed flow over objects such as re-entry objects are found very few. Notable investigations on the re-entry simulations reported are flow over Apollo (Glass et al., 2006; Walpot et al., 2012), Orion (Votta et al., 2013; Palharini, 2014)] and OREX capsule (Moss et al., 1996).

In India, the important mile stone towards re-entry capsule has been due to the Space Recovery Experiment (SRE). It is worth mentioning that the re-entry objects have differences in size as well as shape. The present work is motivated from the curiosity in understanding the effect of the variations in the size and shape in a model of SRE on the different aspects of non-continuum hypersonic flow. Images and important dimensional details of SRE capsule are found from the literature (Harvey et al., 2011). These details are used in the present work to develop the geometrical model of SRE capsule. Major difference of SRE capsule from the capsules of other nations is in the use of a small nose radius (ie. less blunted). To the best of author's knowledge no numerical simulation of SRE model has been reported. Another significance of this work is the usage of an open source computational tool. The methodology of the current work consists of validating dsmcFoam and then apply for computing the flow over a model of the ISRO's SRE capsule. The analysis is performed to find out the heat flux during re-entry corresponding to the conditions of different altitudes and angles of attack. It may be noted that predicted heat flux should not depend considerably on the body surface temperature used as a boundary condition. To verify this hypothesis, the effect of surface temperature distribution on the heat flux is investigated by varying the temperature in the range of 300-2000 K. The minimum value of 300 $\mathrm{K}$ is taken because the atmospheric temperature at $115 \mathrm{~km}$ altitude has a nearly the same value. The maximum value $2000 \mathrm{~K}$ is taken based on the reported value of peak surface temperature (Moss et al., 1996, 2006). In a re-entry process the velocity of capsule changes. It is found from literature (ISRO, 2016) that the maximum speed at re-entry of SRE capsule is $8000 \mathrm{~m} / \mathrm{s}$. Therefore, the free stream velocity of air is kept as $8000 \mathrm{~m} / \mathrm{s}$ for all cases of simulation. Due to the usage of maximum speed of air, heat flux predicted will also be a maximum limiting value. As a part of validation of the DSMC tool, the case of high speed flow over flat plate is analysed and results are compared with reported results (Bird, 1994).

\section{DSMC METHOD}

DSMC method is a particle based sampling method in which one simulated molecule, represents a larger ensemble of molecule. The simulated sample of molecules are tracked through space and time (Bird, 1994). An assumption of the simulation method is that the molecular motion and interaction with the walls are decoupled from intermolecular collisions. The movement of each particle is linear in nature as prescribed by its velocity vector and time-step increment. Reorientation in the particle trajectory occurs as a result of interactions with wall and intermolecular collisions. The particle motion and collision with walls are deterministic and intermolecular collisions have a probabilistic nature. The collision rate is calculated using kinetic theory of gases. The probability of collision between collision partners is simulated using an acceptance rejection test method. The energy exchange between different modes of motion such as transition, rotation and vibration in molecules are included in the collision calculations using Larsen-Borgnakke statistical model (Borgnakke and Larsen, 1975). The macroscopic properties are found out as ensemble average of the properties of particles. The temperature in the flow domain is computed as a weighted average of different modes of energy.

\section{1. dsmcFoam}

OpenFOAM (OpenFOAM, 2016) is an opensource fluid flow solver containing a variety of solvers developed using object oriented $\mathrm{C}++$ libraries. The solvers are developed to handle a number physical problems. The dsmcFoam (Scanlon et al., 2010) is a DSMC solver available in OpenFOAM. From the general description about DSMC method given in section 2, it is clear that the computational requirements of DSMC is different from that of conventional CFD for continuum computations. Common features in conventional CFD and DSMC are on the mesh and time step data. In dsmcFoam, this features for grid and time step are introduced by using the file "fvcfd.h". The features exclusively required for DSMC simulation are introduced using a set of functions and classes through the file "dsmccloud.h". This file redefines the boundary conditions which are used in the conventional CFD to the boundary conditions suitable for molecular dynamics simulation. For example symmetry boundary condition in dsmcFoam performs as specular reflection. A few access functions and submodels included using dsmccloud for DSMC simulations are shown in Table 1.

Table 1 Typical functions and sub models available in dsmcFoam.

\begin{tabular}{|l|l|l|l|}
\hline dsmccloud & \multicolumn{3}{|c|}{ Sub models } \\
\hline $\begin{array}{l}\text { Typical } \\
\text { functions }\end{array}$ & $\begin{array}{l}\text { Inflow } \\
\text { model }\end{array}$ & $\begin{array}{l}\text { Wall iteration } \\
\text { model }\end{array}$ & $\begin{array}{l}\text { Binary } \\
\text { collision model }\end{array}$ \\
\hline move & Free stream & $\begin{array}{l}\text { Maxwellian } \\
\text { Thermal }\end{array}$ & $\begin{array}{l}\text { No Binary } \\
\text { collision }\end{array}$ \\
\hline $\begin{array}{l}\text { buidcell- } \\
\text { occupancy }\end{array}$ & \multirow{2}{*}{ No Inflow } & $\begin{array}{l}\text { Specular } \\
\text { Reflection }\end{array}$ & $\begin{array}{l}\text { Variable Hard } \\
\text { Sphere (VHS) }\end{array}$ \\
\cline { 3 - 4 } calculatefields & & $\begin{array}{l}\text { Mixed Diffusion } \\
\text { Specular }\end{array}$ & $\begin{array}{l}\text { Larsen } \\
\text { Borngnakke VHS }\end{array}$ \\
\hline
\end{tabular}

\subsection{Code Validation}

The open source DSMC code is validated before applying it for the prediction of flow parameters of SRE re-entry model. This is done by simulating flow of nitrogen gas over a horizontal flat plate of $1 \mathrm{~m}$ length. The free stream conditions of the gas has a number density of $10^{20}$ per $\mathrm{m}^{3}$ and temperature of $273 \mathrm{~K}$. The free stream Mach number is taken as 4. The value of free stream Knudsen number calculated using length as characteristic dimension of 0.0143 . Though this value of Kn belongs to the continuum range, features of the flow leads to local rarefaction. The molecular properties of nitrogen gas and boundary conditions used are tabulated in table 2 and table 3 , respectively. The computational results for the problem is available for comparison in Bird (1994).

Table 2 Molecular properties of $N_{2}$ used for high speed flow over flat plate, where $T_{\text {ref }}$ is the reference temperature, $\omega$ is viscosity index, $\mathrm{d}$ is molecular diameter and $\mathrm{m}$ is molecular mass

\begin{tabular}{|c|c|c|c|}
\hline$T_{r e f}$ & $\omega$ & $d(\mathrm{~m})$ & $m(\mathrm{~kg})$ \\
\hline 273 & 0.74 & $4.17 \times 10^{-10}$ & $4.65 \times 10^{-26}$ \\
\hline
\end{tabular}

Computation is carried out using a time step of $4 \times 10^{-6} s$ and simulation is done up to $1 \mathrm{~s}$. The contours of flow angle and temperature from the present investigation and that reported by Bird (1994) are shown in red 
Table 3 Boundary conditions for high speed flow over flat plate, where $\mathrm{M}$ is the Mach number, $\mathrm{V}$ is the velocity, $\mathrm{T}$ is the temperature, $\mathrm{n}$ is the number density and $\alpha$ is the free stream condition.

\begin{tabular}{|c|c|c|c|c|}
\hline$M_{\infty}$ & $V_{\infty}(m / s)$ & $T_{\infty}(K)$ & $T_{\text {wall }}(K)$ & $n_{\infty}\left(m^{-3}\right)$ \\
\hline 4 & 1347.6 & 273 & 500 & $1 \times 10^{20}$ \\
\hline
\end{tabular}

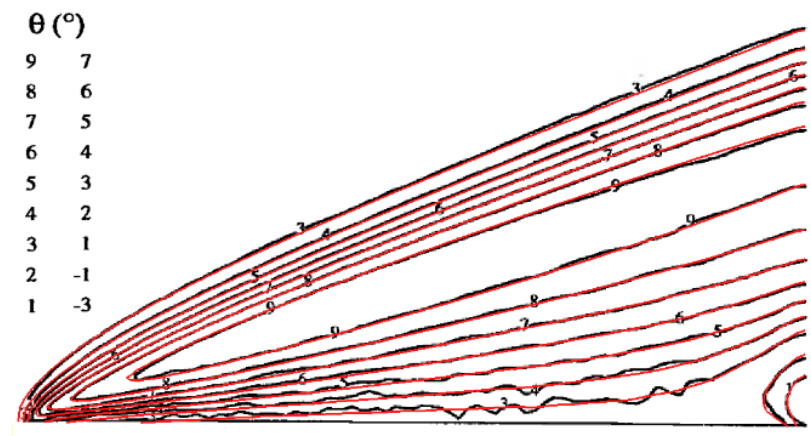

Fig. 1 Contours of flow angle over flat plate (red lines-from dsmcFoam, black lines-from Bird (1994)).

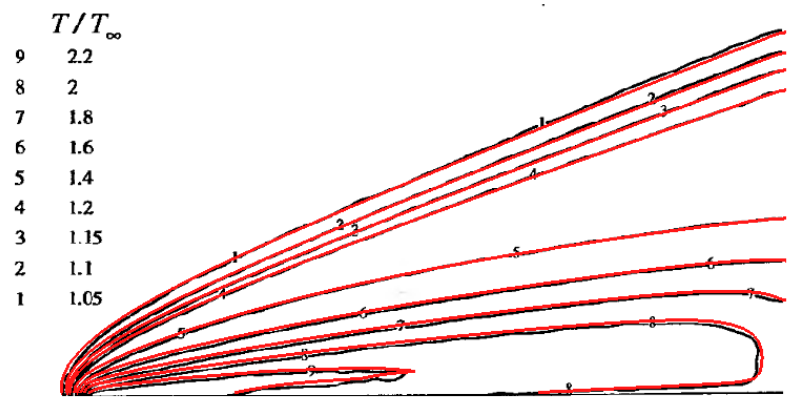

Fig. 2 Temperature contours over flat plate (red lines-from dsmcFoam, black lines-from Bird (1994)).

and black colored lines, respectively, in figure 1 and 2 . The surface properties such as coefficient of pressure $(\mathrm{Cp})$ and heat transfer coefficient (Ch ) are also compared with the results of Bird (1994) in figures 3 and 4. A close agreement of flow characteristics captured by dsmcFoam and those of Bird is clearly demonstrates the validity of molecular model as well as the implementation of the DSMC method.

With the confidence gained by solving the validation problem with $M_{\infty}=$ 4 , an attempt is made to use dsmcFoam for the simulation of flow past SRE model with $M_{\infty}$ greater than 25 . It may be noted that with a large value of difference in the free stream Mach number the validity of the molecular model and dsmcFoam can be assessed from the results of simulations.

\section{SRE MODEL}

The model of re-entry capsule has a spherically blended cone configuration having a base diameter of $2.0 \mathrm{~m}$, height of $2.1 \mathrm{~m}$ and nose radius of $0.5 \mathrm{~m}$. The shape and dimensions of capsule shown in figure 5 have been taken from the data available in the literature (Harvey et al., 2011).

\subsection{Computational Domain and Mesh}

Three dimensional flow domain considered for the study has $6 \mathrm{~m}$ length upstream of nose and $15 \mathrm{~m}$ length in the downstream of rear end of the body. The surface mesh used over body, and volume mesh developed for the simulation are shown in figures 6 and 7 respectively. This mesh has been developed using ANSYS ICEM 14.0, which is imported to OpenFOAM using fluent3DMeshToFoam tool. In ICEM CFD, block meshing

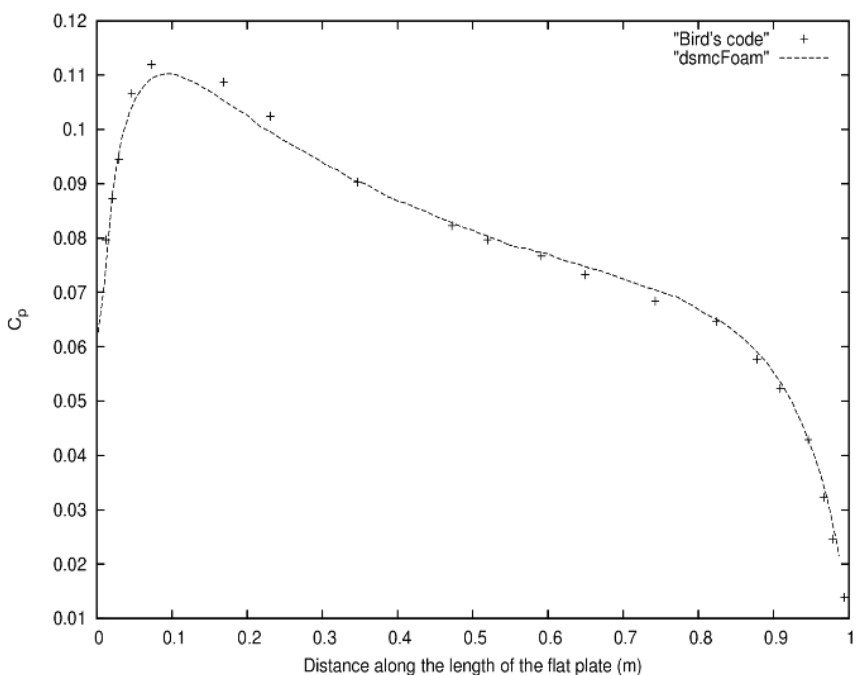

Fig. 3 Comparison of coefficient of pressure $(\mathrm{C} \mathrm{p})$ over the flat plate.

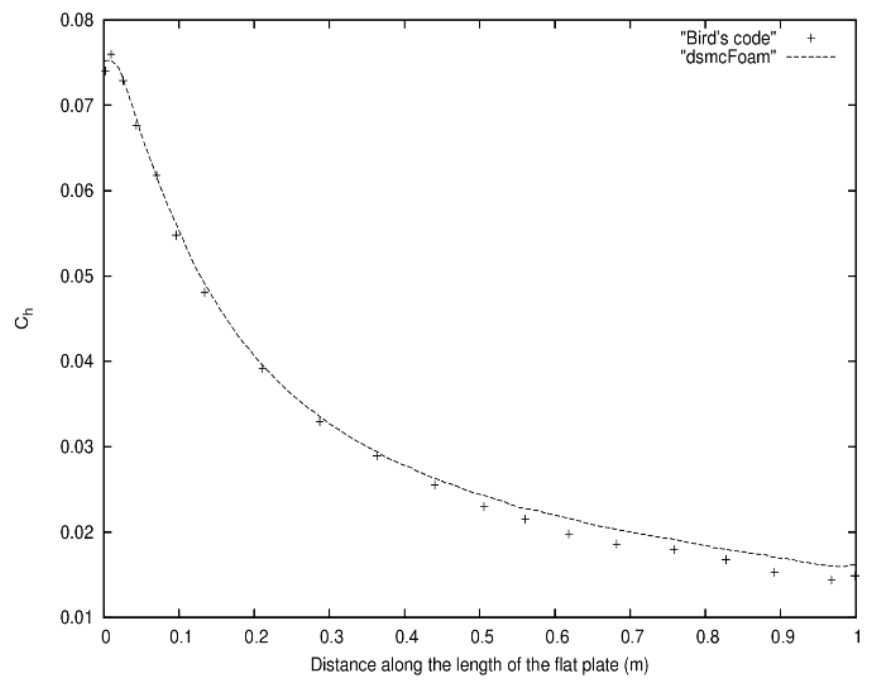

Fig. 4 Comparison of heat transfer coefficient $(\mathrm{C} \mathrm{h})$ over the flat plate.

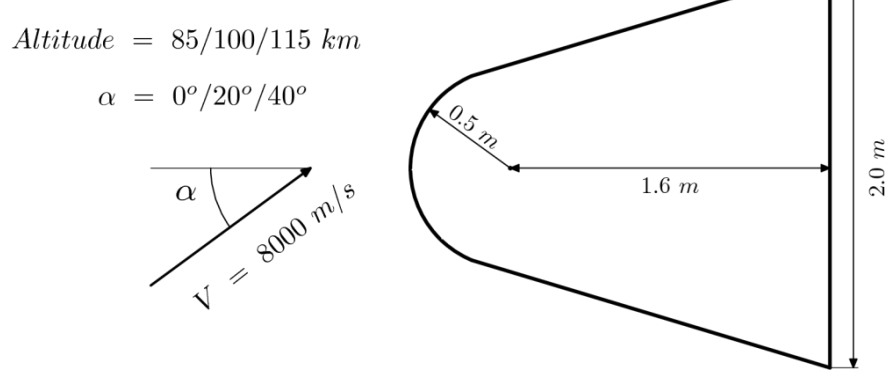

Fig. 5 Important dimensions of model of SRE re-entry object. 
is applied to generate unstructured hexahedral cells. The mesh consists of a total of 4.0 million hexahedral cells.
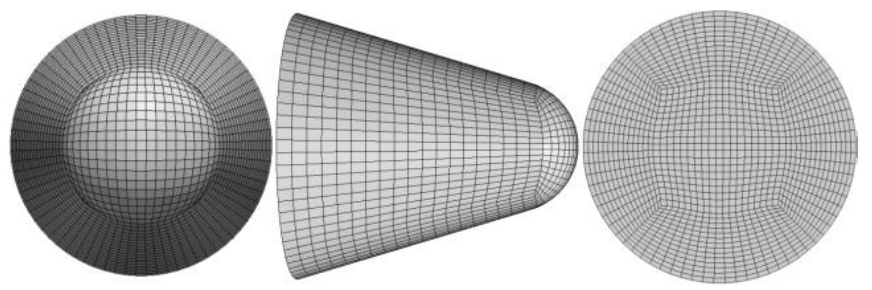

Fig. 6 The surface mesh on SRE model.

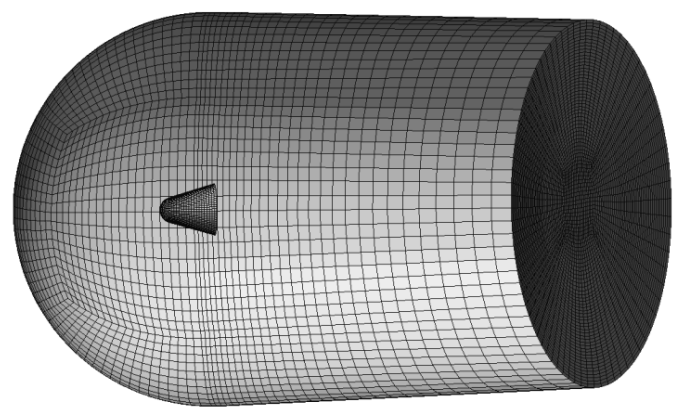

Fig. 7 Flow domain for the simulation of hypersonic flow over SRE model.

\subsection{Boundary Conditions and Molecular Property}

During re-entry, SRE travels down from $120 \mathrm{~km}$ to $14 \mathrm{~km}$ altitude. In this work steady state DSMC simulations are carried out for conditions corresponding to three different altitudes of $85 \mathrm{~km}, 100 \mathrm{~km}$ and $115 \mathrm{~km}$. Composition of the gas and free stream temperature at various altitudes used for the analysis are taken from reference NASA (2016) and are tabulated in table 4 . The values of free stream Knudsen numbers are calculated by taking the nose diameter as characteristic dimension for re-entry altitudes of $85 \mathrm{~km}, 100 \mathrm{~km}$ and $115 \mathrm{~km}$ are $0.01,0.12$ and 2.0, respectively. The

Table 4 The free stream number density and temperature conditions used as boundary conditions for the simulation of SRE model obtained from NASA's MSIS-E-90 atmospheric model (NASA, 2016).

\begin{tabular}{|c|c|c|c|c|}
\hline \multirow{2}{*}{$\begin{array}{l}\text { Altitude, } \\
\mathrm{km}\end{array}$} & \multicolumn{3}{|c|}{ Number density $\times 10^{-17}, \mathrm{~m}^{-3}$} & \multirow{2}{*}{$\begin{array}{l}\text { Free stream } \\
\text { temperature, } K\end{array}$} \\
\cline { 2 - 4 } & $\mathrm{N}_{2}$ & $\mathrm{O}_{2}$ & $\mathrm{O}$ & 208.9 \\
\hline 85 & 1728.01 & 458.31 & 0.39 & 165.1 \\
\hline 100 & 95.98 & 22.82 & 4.85 & 297.1 \\
\hline 115 & 6.32 & 1.08 & 1.23 & \\
\hline
\end{tabular}

re-entry of velocity is taken as $8000 \mathrm{~m} / \mathrm{s}$. The gas considered is a mixture of Nitrogen and Oxygen. The molecular properties of species in the free stream flow are given in table 5 .

Simulation of flow requires the surface temperature of the body as a boundary condition. The body surface temperature is unknown. In this work, a parametric study is first carried out by varying surface body temperature to check the dependence of surface temperature on the predicted heat flux. The surface temperature of the body has been varied as 300 , $500,1000,1500$ and $2000 \mathrm{~K}$. The variation of predicted surface heat flux as a function of axial distance from the nose of the body is presented

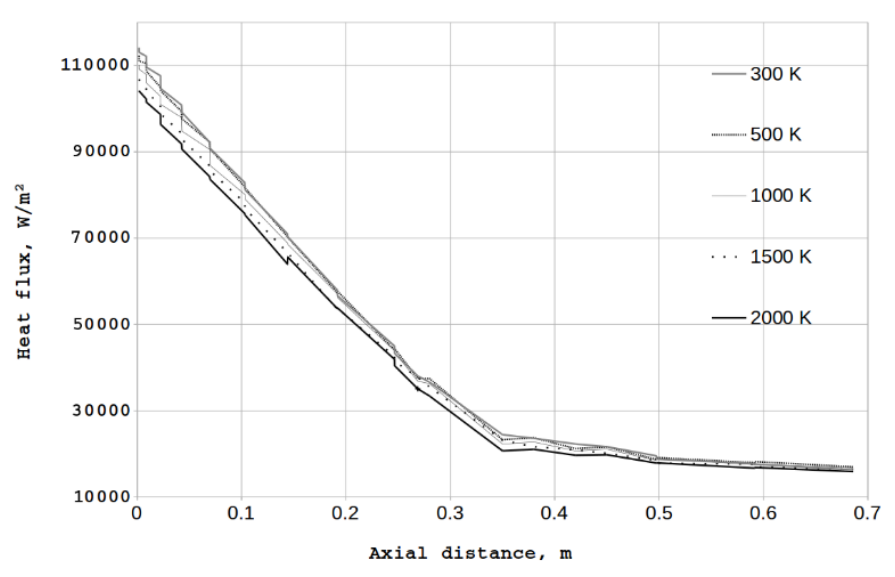

Fig. 8 Variation of heat flux over the body surface at $100 \mathrm{~km}$ altitude and $\alpha=0^{\circ}$ for different surface temperature boundary condition.

in figure 8. It shows that the surface heat flux distribution is altered only very little by variations of surface temperature used as boundary condition. As the maximum percentage deviation is only $8 \%$, a surface temperature of $300 \mathrm{~K}$ is applied for all the remaining simulations. The flow consisting of diatomic molecules, rotational relaxation is enabled by using binary collision model as "LarsenBorgnakkeVariableHardSphere" option in dsmcFoam. The coefficient of rotational relaxation number for energy exchange is taken as 5. This value is entered through the variable "relaxationCollisionNumber" corresponding to the reference temperature of $273 \mathrm{~K}$. Over the SRE capsule surface, the Maxwellian thermal model is used to prescribe a constant wall temperature. Simulations have been carried out for three values of angle of attack $\alpha$ of the re-entry capsule such as $0^{\circ}, 20^{\circ} \& 40^{\circ}$.

Table 5 Molecular properties of free stream gas at a $T_{r e f}$ of $273 \mathrm{~K}$, where $\omega$ is viscosity index, $\mathrm{d}$ is molecular diameter, $\mathrm{m}$ is molecular mass, idof is the number of internal degree of freedom.

\begin{tabular}{|c|c|c|c|c|}
\hline Molecule & $\omega$ & $d_{\text {ref }} \times 10^{10}, \mathrm{~m}$ & $\mathrm{~m} \times 10^{27}, \mathrm{~kg}$ & idof \\
\hline $\mathrm{N}_{2}$ & 0.74 & 4.07 & 46.50 & 2 \\
\hline $\mathrm{O}_{2}$ & 0.77 & 3.96 & 53.10 & 2 \\
\hline $\mathrm{O}$ & 0.80 & 3.00 & 26.50 & 1 \\
\hline
\end{tabular}

\subsection{Computational Details}

Accuracy of DSMC simulations depends on cell size, time step, number of simulated molecules and number of sampling time steps used for macroscopic averaging. The cell size and time step should be less than or equal to the mean free path and mean collision time, respectively. The simulated number of molecules must be enough for the computation of collisions. Time steps sampled for macroscopic property evaluation should be large enough to reduce statistical error. By keeping these considerations, a total number of initial simulated molecules of approximately 2.0 million and time step of $1 \times 10^{-6} s$ are taken for the case of 85 $\mathrm{km}$ altitude. The simulation is carried out using parallel computation, and has taken about 150 hours of computer run time for the simulation for a total physical time of $0.1 \mathrm{~s}$. The computer used is Dell PowerEdge M620 blade server with Intel Xeon processor E5-2600 2.0 GHz-48 processor.

\section{RESULTS AND DISCUSSION}

The results presented here consists of variations of surface flow properties, flow domain temperature and density distributions. The variation of surface flow properties presented are that of pressure, shear stress, heat flux, slip velocity and temperature jump as a function of distance along 


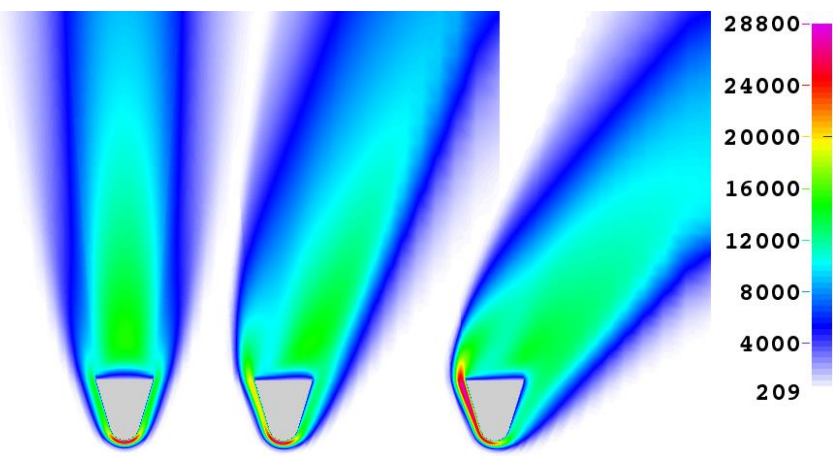

(a) Altitude $=85 \mathrm{~km}$
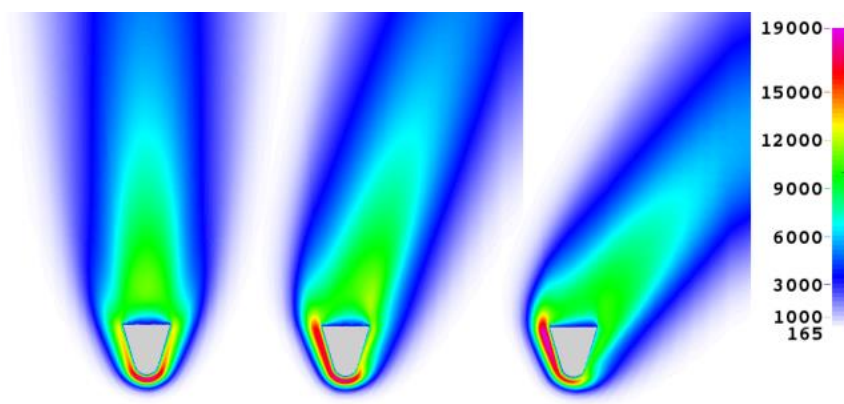

(b) Altitude $=100 \mathrm{~km}$

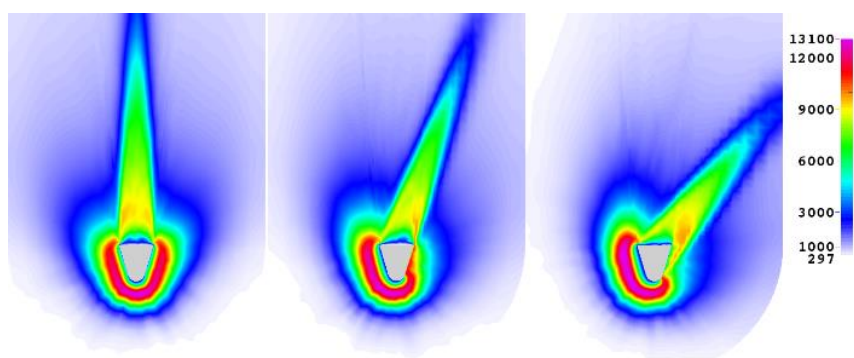

(c) Altitude $=115 \mathrm{~km}$

Fig. 9 The contours of temperature $(\mathrm{K})$ over the symmetry plane of flow domain for different altitudes and angles of attack.

the axis from the leading edge. The integral quantities reported are total heat entering into the body, pressure drag, and frictional drag. These quantities have been computed by integrating their flux values such as the heat flux, surface pressure and surface shear stress, respectively. In the following discussion of results, the portion of the surface of the body facing the free stream flow and the remaining portions are referred as windward and leeward side, respectively. These two surfaces are different for flow with a non-zero angle of attack only.

\subsection{Contours of Temperature and Density}

Figure 9 shows the contours of overall temperature along the symmetry plane of flow domain for different altitude conditions and angles of attack. With increase of angle of attack, the shock structure changes and maximum temperature is found to shift towards the windward side. A clear difference in the shock envelope with the change of angle of attack can be observed. The after body flow consist of a tail of high temperature zone, which is extending to downstream of flow. The peak value of temperature in the domain for altitudes 85,100 and $115 \mathrm{~km}$ are obtained as $28,800,19,000$ and $13,100 \mathrm{~K}$. With increase of altitude, the value of temperature reduces and its location moves away from the surface of the body. This type of temperature distribution, in conjunction with relatively low values of number density do not cause a rate of chemical reaction that

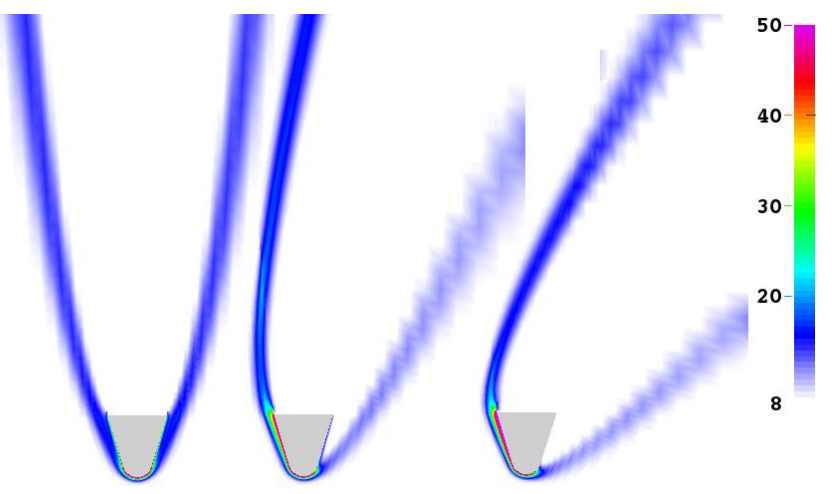

(a) Altitude $=85 \mathrm{~km}$

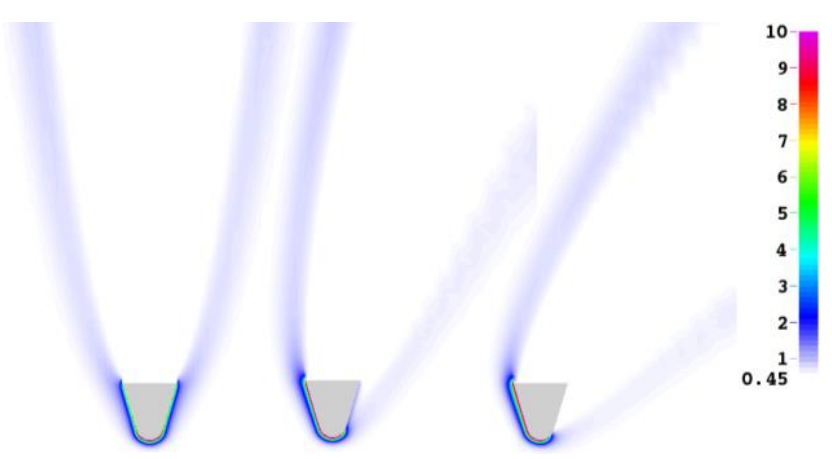

(b) Altitude $=100 \mathrm{~km}$
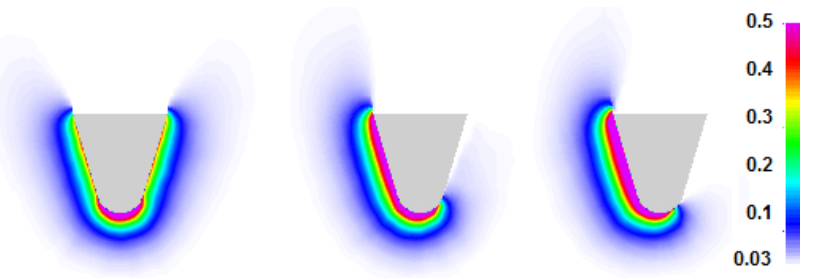

(c) Altitude $=115 \mathrm{~km}$

Fig. 10 The contours of density $\times 10^{6}\left(\mathrm{~kg} / \mathrm{m}^{3}\right)$ over the symmetry plane of flow domain for different altitudes and angles of attack.

can lead to significant variations in the surface properties at higher altitudes such as 100 and $115 \mathrm{~km}$ (Scanlon et al., 2015). However, changes due to chemical reaction corresponding to re-entry condition from altitude $85 \mathrm{~km}$ should not be disregarded and necessary modification of the molecular model may be required for the analysis of hypersonic flow in dense atmospheric conditions such as those at $85 \mathrm{~km}$ altitude. Figure 10 shows contours of density over the symmetric plane of flow domain. It shows that the distribution of gas molecules is more in the vicinity of reentry capsule and shock. The density is very less behind the capsule and downstream of the bow shock. At higher altitudes weak diffused shock having larger thickness is found.

\subsection{Stagnation Line Temperature Variation}

Figure 11 shows the variation of translational, rotational and overall temperatures along the outward normal to forward stagnation point, for the three altitudes such as 85,100 and $115 \mathrm{~km}$ at $\alpha=0^{\circ}$. The peak temperature rise occurs at a certain distance ahead of the nose due the action of the bow shock. The shock standoff distance is measured as the distance in the normal direction from the nose, where the temperature of the gas is maximum. The values of shock stand-off distance for altitudes 85, 100 and $115 \mathrm{~km}$ are $0.065,0.12$ and $0.55 \mathrm{~m}$, respectively. With decrease in altitude, the flow becomes denser and results in thin strong shock and 
the object undergoes high aero-thermodynamic heating. This causes an increase of rotational mode of temperature at lower altitudes compared to higher altitudes. At the forward stagnation point, a maximum overall temperature of approximately $2000 \mathrm{~K}$ has been computed for all the altitude conditions.

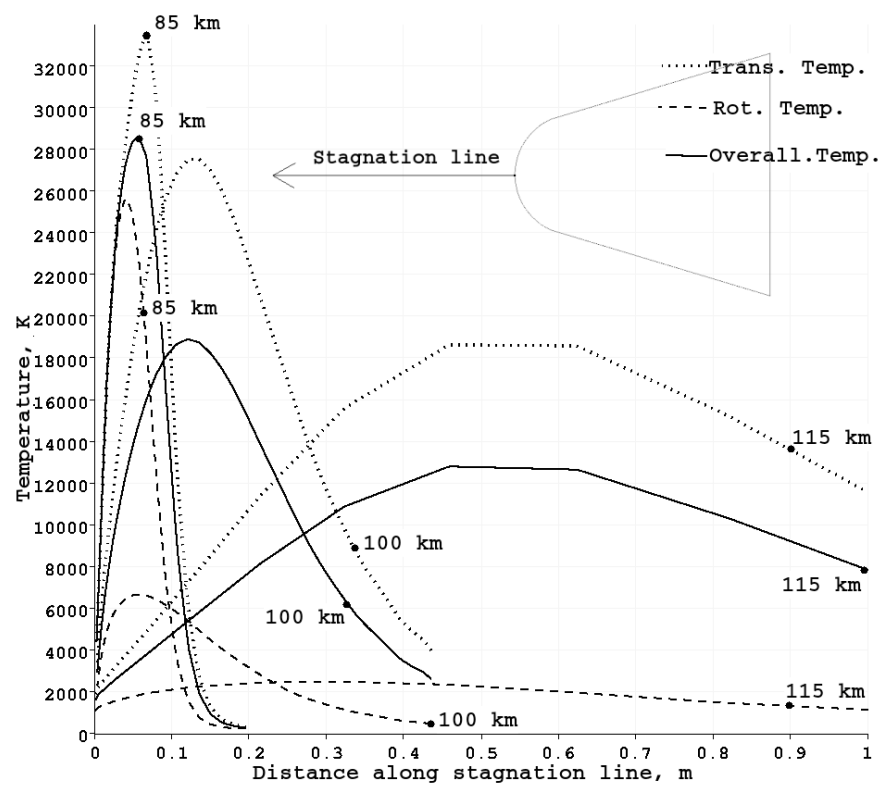

Fig. 11 Distribution of translational, rotational and overall temperature variation over the stagnation line for different altitude at angle of attack of zero degree.

\subsection{Surface Heat Flux}

The surface heat flux over the model and the total heat entering the body for different angles of attack are tabulated in table 6. The magnitude of the peak heat flux increases with reduction of altitude. In all the cases, the peak heat flux is found to occur at the forward stagnation point. The highest value for the peak heat flux on the body is found to occur for the lowest altitude case of $85 \mathrm{~km}$ and angle of attack equal to zero degree. The variations of heat flux over the body surface as a function of axial distance along the middle symmetry plane is shown in figure 12. For angle of attack $=0$, the curves on the windward side and leeward side are same. At angle of attack $\left(\alpha=40^{\circ}\right)$, the location of the peak heat flux move over the windward side due to shifting of the stagnation point. The magnitude of peak heat flux reduces slightly with increases of angle of attack. This is due to the effect of increases of radius of curvature at the point of stagnation. A comparison of present prediction of peak

Table 6 Peak surface heat flux $\left(q_{\text {peak }}\right)$ and total heat $\left(q_{\text {total }}\right)$ over the body surface at different altitudes and angles of attack, $x$ is the axial distance $(\mathrm{m})$ from nose end.

\begin{tabular}{|c|l|r|r|r|}
\hline Value & Altitude & \multicolumn{1}{|c|}{$\alpha=0^{\circ}$} & $\alpha=20^{\circ}$ & $\alpha=40^{\circ}$ \\
\hline \multirow{2}{*}{$\begin{array}{c}q_{\text {peak }} \\
\left(\mathrm{kW} / \mathrm{m}^{2}\right)\end{array}$} & $85 \mathrm{~km}$ & 1040.30 & 977.02 & 963.12 \\
\cline { 2 - 5 } & $100 \mathrm{~km}$ & 114.01 & 108.03 & 106.01 \\
\cline { 2 - 5 } & $115 \mathrm{~km}$ & 9.91 & 9.45 & 9.19 \\
\hline \multirow{2}{*}{$\begin{array}{c}\text { total } \\
(\mathrm{kW})\end{array}$} & $85 \mathrm{~km}$ & 1099.01 & 1494.50 & 1776.91 \\
\cline { 2 - 5 } & $100 \mathrm{~km}$ & 194.70 & 214.71 & 240.71 \\
\cline { 2 - 5 } & $115 \mathrm{~km}$ & 25.41 & 25.12 & 27.62 \\
\hline
\end{tabular}

heat flux with other re-entry capsules at approximately similar conditions and altitude is given in the table 7. The value of peak heat flux for the SRE model is more than that of ORION and OREX capsules. The reason

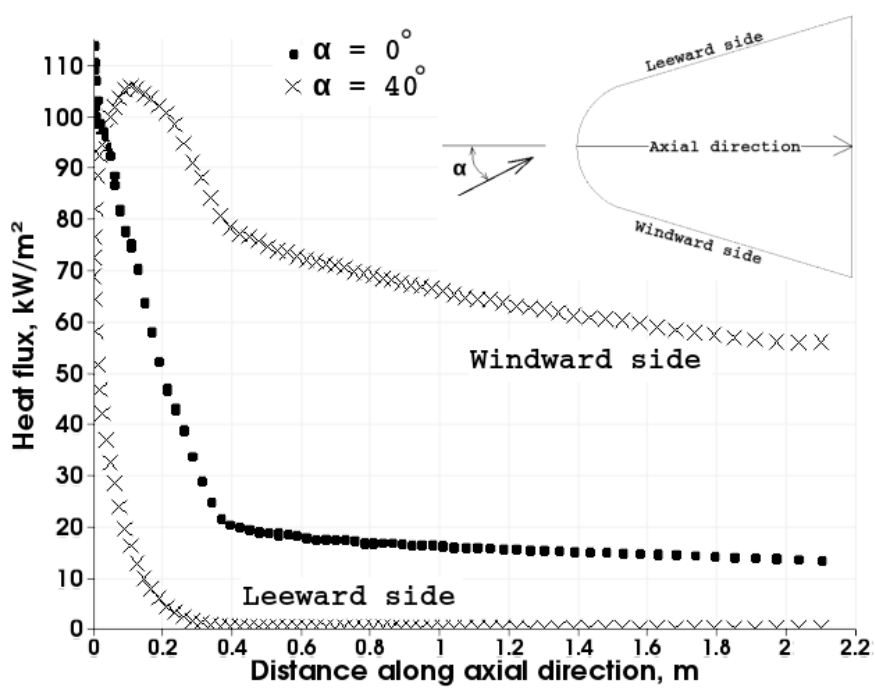

Fig. 12 Distribution of heat flux along the symmetry plane of free stream flow over the body surface for different Îś values at $100 \mathrm{~km}$ altitude.

Table 7 Comparison of peak surface heat flux $\left(q_{\text {peak }}\right)$ of SRE model at zero angle of attack with other re-entry capsules with approximately similar boundary conditions.

\begin{tabular}{|l|l|l|l|}
\hline \multirow{2}{*}{$\begin{array}{c}\text { Altitude, } \\
\mathrm{km}\end{array}$} & \multicolumn{3}{|c|}{$q_{\text {peak }}\left(\mathrm{kW} / \mathrm{m}^{2}\right)$} \\
\cline { 2 - 4 } & \multicolumn{1}{|c|}{ SRE } & ORION & OREX \\
\hline 100 & 114.01 & 56.00 & 53.30 \\
\hline 115 & 9.91 & 5.01 & 7.90 \\
\hline
\end{tabular}

for this difference is attributed to the higher nose radius of ORION and OREX capsule.

\subsection{Surface Pressure}

The values of peak pressure and pressure drag over the body for different cases of altitudes and angle of attack are given in table 8 . The magnitude of the peak pressure is highest for the lowest altitude. This is due to the higher value of number density. In all the cases, the peak pressure is obtained to occur at the forward stagnation point. The highest peak pressure on the body is found for the lowest altitude of $85 \mathrm{~km}$ altitudes and for zero degree angle of attack. The variations of pressure over the body surface as a function of axial distance along the middle symmetry plane is shown in figure 13. At angle of attack $\alpha=40^{\circ}$, the peak pressure of $39.3 \mathrm{~Pa}$ occurring at the leading edge undergoes a sudden drop and becomes equal to 27 at an axial distance of $0.4 \mathrm{~m}$. Thereafter the pressure remains more or less a constant. The peak value of pressure is found to be only slightly altered by the change in angle of attack from 0 to 40 degree.

Table 8 The peak surface pressure $\left(P_{\text {peak }}\right)$ and total pressure drag $\left(P_{\text {drag }}\right)$ over the body surface at different altitudes and angles of attack, $x$ is the axial distance $(\mathrm{m})$ from nose end.

\begin{tabular}{|c|l|r|r|r|}
\hline Value & Altitude & \multicolumn{1}{|c|}{$\alpha=0^{\circ}$} & \multicolumn{1}{|c|}{$\alpha=20^{\circ}$} & $\alpha=40^{\circ}$ \\
\hline \multirow{2}{*}{$P_{\text {peak }}$} & $85 \mathrm{~km}$ & 656.02 & 624.10 & 617.31 \\
\cline { 2 - 5 }$(P a)$ & $100 \mathrm{~km}$ & 39.31 & 37.42 & 36.8 \\
\cline { 2 - 5 } & $115 \mathrm{~km}$ & 2.67 & 2.55 & 2.48 \\
\hline \multirow{2}{*}{$\begin{array}{c}P_{\text {drag }} \\
(N)\end{array}$} & $85 \mathrm{~km}$ & 762.22 & 1091.01 & 1501.20 \\
\cline { 2 - 5 } & $100 \mathrm{~km}$ & 53.32 & 66.21 & 87.30 \\
\cline { 2 - 5 } & $115 \mathrm{~km}$ & 3.81 & 4.44 & 5.84 \\
\hline
\end{tabular}




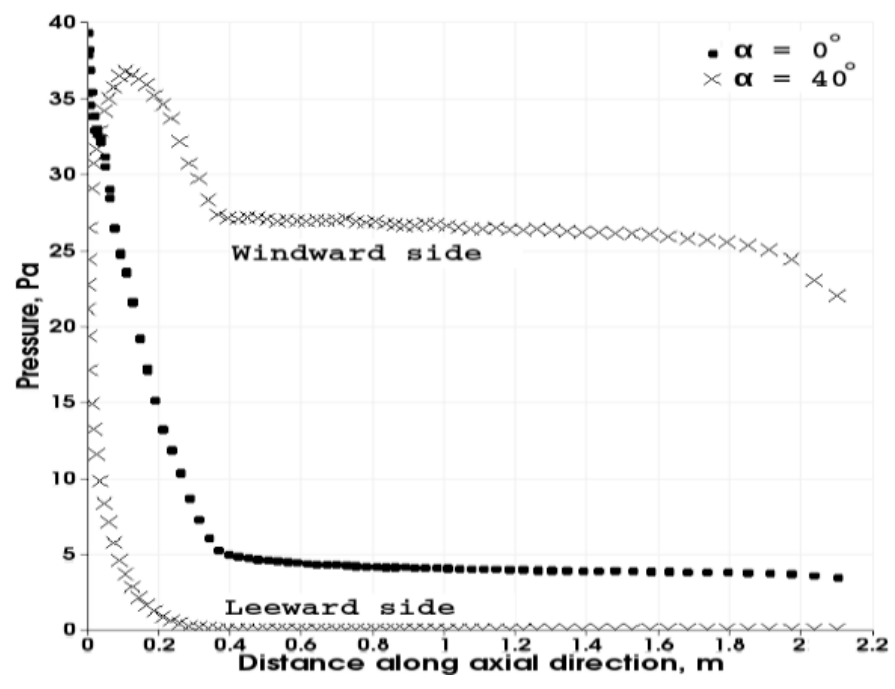

Fig. 13 Distribution of surface pressure along the symmetry plane of free stream flow the body surface for different angles of attack at 100 $\mathrm{km}$ altitude.

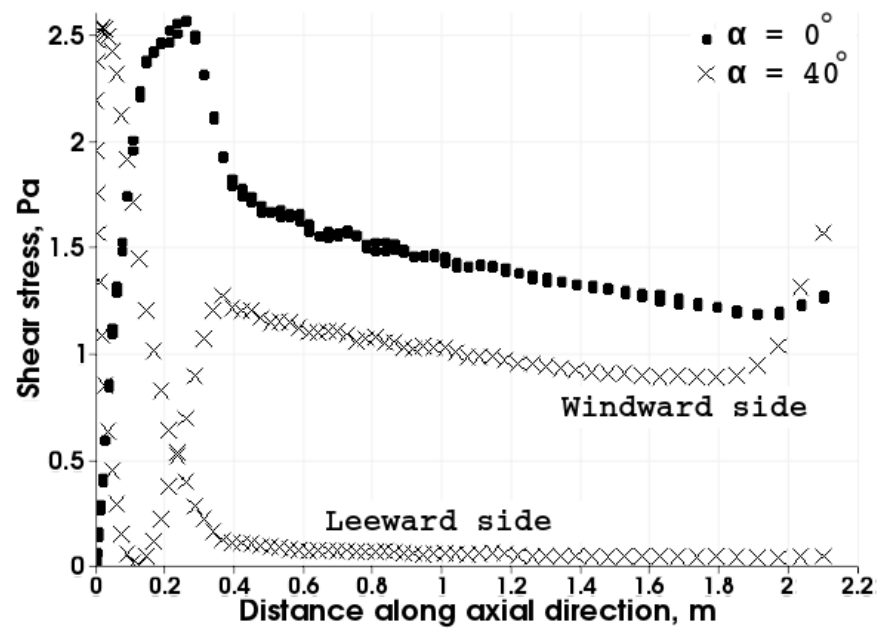

Fig. 14 Distribution of shear stress along the symmetry plane of free stream flow over the body surface for different angles of attack at $100 \mathrm{~km}$ altitude.

\subsection{Surface Shear Stress}

The values of peak shear stress and frictional drag over the body for different altitudes and angles of attack are shown in table 9. The magnitude of the peak shear stress increases with decreases of altitude. The highest value for the peak shear stress on the body is found for the lowest altitude of $85 \mathrm{~km}$ and for highest angle of attack of 40 degree. At higher altitudes change in angle of attack does not make significant difference in shear stress. This is due to the low value of number density. The variations of shear stress over the body surface as a function of axial distance along the middle symmetry plane is shown in figure 14 .

\subsection{Slip Velocity}

Figure 15 shows the variation of slip velocity over the body surface. No slip condition is observed at the leading edge of the body at all conditions of altitude as well as angles of attack. The slip velocity increases along the surface, reaches a maximum and then reduces slightly towards the back end of the body for zero angle of attack. Due to the abrupt geometry change at the back end of the body, a small fraction of the particles fall down from its corner and the local number density on the surface close
Table 9 The peak shear stress $\left(\tau_{\text {peak }}\right)$ and friction drag $\left(\tau_{\text {drag }}\right)$ over the body surface at different altitudes and angle of attack, $x$ is the axial distance $(\mathrm{m})$ from nose end.

\begin{tabular}{|c|l|r|r|r|}
\hline Value & Altitude & $\alpha=0^{\circ}$ & $\alpha=20^{\circ}$ & $\alpha=40^{\circ}$ \\
\hline \multirow{2}{*}{$\tau_{\text {peak }}$} & $85 \mathrm{~km}$ & 7.98 & 10.50 & 11.81 \\
\cline { 2 - 5 }$(P a)$ & $100 \mathrm{~km}$ & 2.64 & 2.62 & 2.62 \\
\cline { 2 - 5 } & $115 \mathrm{~km}$ & 0.45 & 0.45 & 0.45 \\
\hline \multirow{2}{*}{$\tau_{\text {drag }}(N)$} & $85 \mathrm{~km}$ & 28.02 & 36.61 & 3.21 \\
\cline { 2 - 5 }$(100 \mathrm{~km}$ & 13.80 & 12.10 & 8.60 \\
\cline { 2 - 5 } & $115 \mathrm{~km}$ & 3.01 & 2.30 & 1.75 \\
\hline
\end{tabular}

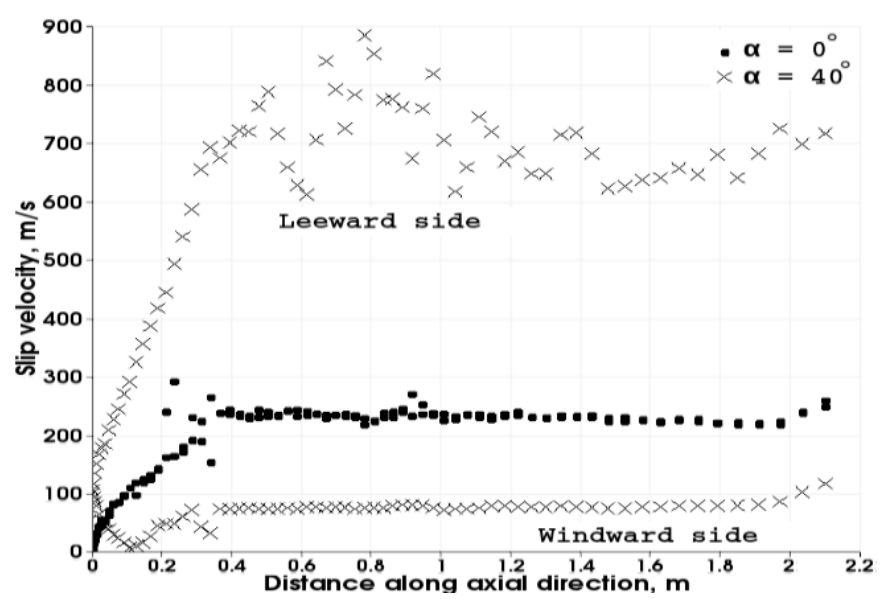

Fig. 15 Distribution of slip velocity along the symmetry plane of free stream flow over the body surface for different angles of attack at $100 \mathrm{~km}$ altitude.

to the back end of the body drops locally. This local drooping of number density causes an increase of slip velocity close to the back end of the body. With increase of angle of attack the maximum value of slip velocity in the leeward side increases. In the leeward side slip velocity is as high as $800 \mathrm{~m} / \mathrm{s}$ for angle of attack of 40 degree. The windward side is subjected to more collisions with gas molecules, so here slip velocity is comparatively less. The slip velocity in the windward side for $\alpha=40^{\circ}$ is as low as $90 \mathrm{~m} / \mathrm{s}$. Fluctuations seen in the predicted values of slip velocities in the leeward side in figure 15 is due to the random molecular phenomena.

\subsection{Temperature Jump}

The distribution of temperature jump over the body surface is given in figure 16 , which shows that the variation is similar to that of slip velocity as found in figure 15 . Higher slip velocity occurs at the regions of low number density due to the effect of rarefaction. Less number of particles present in the high slip regions are subjected to minimum energy interaction by surface collision leading to a larger temperature jump. Even though temperature jump is maximum, the total energy transfer to the walls is not maximum due to lower number of particle interactions. Due to this reason, the regions of maximum temperature jump do not coincide with regions of maximum heat flux. Further, the heat transfer coefficient in these high speed regions is greatly reduced due to low heat flux and high temperature jump. Temperature jump is found to be as high as 3000 $\mathrm{K}$ for altitude $100 \mathrm{~km}$, with a minimum value of $950 \mathrm{~K}$ occurring at the windward side.

\section{CONCLUSIONS}

The present work has been able to provide a validation for the DSMC implementation in dsmcFoam for high speed flow of diatomic Nitrogen 


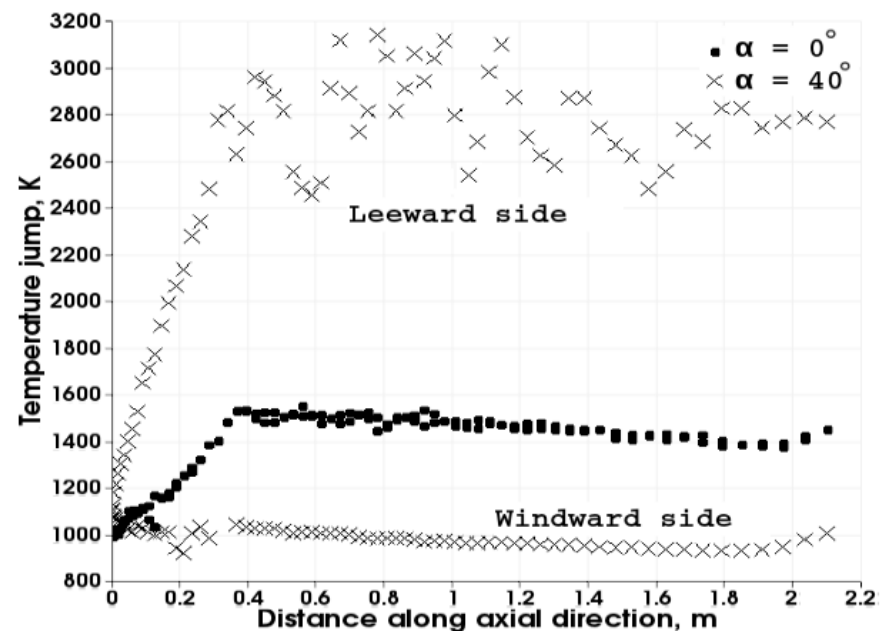

Fig. 16 Distribution of temperature jump along the symmetry plane of free stream flow over the body surface for different angles of attack at $100 \mathrm{~km}$ altitude.

gas over a flat plate. Further, the study has been able to establish the applicability of dsmcFoam to hypersonic flow over a re-entry object, SRE capsule at different altitudes and angles of attack. Based on the field temperature and available data from literature, it is concluded that the surface properties predicted using molecular model consisting of binary collision interactions with boundaries and other molecules is valid for high free stream Knudsen number corresponding to 100 and $115 \mathrm{~km}$ altitudes. Predictions for the lowest Knudsen number analyzed, corresponding to $85 \mathrm{~km}$ altitude show temperature values capable of resulting into increased rate endothermic chemical reactions that may be able to significantly alter the predicted surface properties. Extending the molecular model by adding vibrational mode of energy exchange and chemical reaction should prove a reduction of maximum temperature in the flow field and accurate prediction of surface properties even for low altitude conditions. This is considered as a scope for future work. Another important conclusion from the present investigation is on the effect of wall temperature boundary condition on the simulated properties. It is concluded that heat flux is affected very little by variation of surface temperature in the interval [300-2000 K].

A brief summary of the important results obtained from the study is given below.

The lower nose radius of present model results in high value for the peak heat flux compared to the other re-entry models. The location of peak values for heat flux and surface pressure are at the forward stagnation point, but the peak temperature at low number dense regions having minimum energy interaction with the surfaces. For zero angle of attack, the peak value of heat flux for 85,100 and $115 \mathrm{~km}$ altitudes are $1040.30,114.01 \& 9.91 \mathrm{~kW} / \mathrm{m}^{2}$, respectively. For $85 \mathrm{~km}$ altitude, a sharp thin bow shock wave is formed in front of the capsule. More diffusive bow shock waves are obtained for altitudes $100 \mathrm{~km}$ and $115 \mathrm{~km}$. Increase of angle of attack changes the shape of shock envelop, which largely influences the surface properties.

\section{REFERENCES}

Anderson, J.D., 2006, Hypersonic and High Temperature Gas Dynamics, American Institute of Aeronautics and Astronautics.

http://dx.doi.org/10.2514/4.861956.

Bird, G.A., 2007, "Sophisticated DSMC, Notes from a Short Course," DSMC07 Conference, Santa Fe, USA.

Bird, G.A., 1994, Molecular Gas Dynamics and the Direct Simulation of
Gas Flows, Oxford Science Publications.

Borgnakke, C., and Larsen, P.S., 1975, "Statistical Collision Model for Monte Carlo Simulation of Polyatomic Gas Mixture," Journal of computational Physics, 18(4), 405-420.

http://dx.doi.org/10.1016/0021-9991(75)90094-7.

Cercignani, C., 2000, Rarefied Gas Dynamics: from Basic Concepts to Actual Calculations, Cambridge University Press.

Chen, G., and Boyd, I.D., 1996, "Statistical Error Analysis for the Direct Simulation Monte Carlo Technique," Journal of Computational Physics, 126(2), 434-448.

http://dx.doi.org/10.1006/jcph.1996.0148.

Gallis, M.A., Torczynski, J., Rader, D., and Bird, G.A., 2009, "Convergence Behavior of a New DSMC Algorithm," Journal of Computational Physics, 228(12), 4532-4548.

http://dx.doi.org/10.1016/j.jcp.2009.03.021.

Glass, C.E., Moss, J.N., and Greene, F.A., 2006, "DSMC Simulations of Apollo Capsule Aerodynamics for Hypersonic Rarefied Conditions," 9 th AIAA/ASME Joint Thermo physics and Heat Transfer Conference.

Harvey, B., Smid, H.H., and Pirard, T., 2011, Emerging Space Powers: the New Space Programs of Asia, the Middle East and South-America, Springer Science \& Business Media.

ISRO, 2016, "ISRO Official Website," http://www.isro.gov.in/update/22-jan-2007/space-capsule-successfullyrecovered.

LeBeau, G.J., Boyles, K.A., and Lumpkin, F., 2003, "Virtual Sub-cells for the Direct Simulation Monte Carlo Method," AIAA paper, 1031, 2003.

Moss, J.N., Boyles, K.A., and Greene, F.A., 1996, "DSMC Simulations of OREX Entry Conditions," NASA-TM-111621.

Moss, J.N., Boyles, K.A., and Greene, F.A., 2006, "Orion Aerodynamics for Hypersonic Free Molecular to Continuum Conditions," 14th AIAA international space planes and hypersonic systems and technologies conference.

NASA, 2016, "MSIS-E-90 Atmosphere Model, NASA," http://omniweb.gsfc.nasa.gov/vitmo/msis_vitmo.html.

OpenFOAM, 2016, “OpenFOAM Offcial Wesite," http://www.openfoam.com/.

Palharini, R.C., 2014, Atmospheric Re-entry Modelling Using an Opensource DSMC Code, Ph.D. thesis, University of Strathclyde.

Scanlon, T.J., White, C., Borg, M.K., Palharini, R.C., Farbar, E., Boyd, I.D., Reese, J.M., and Brown, R.E., 2015, "Open-source Direct Simulation Monte Carlo Chemistry Modeling for Hypersonic Flows," AIAA Journal.

Scanlon, T., Roohi, E., White, C., Darbandi, M., and Reese, J., 2010, “An Open-source, Parallel DSMC Code for Rarefied Gas Flows in Arbitrary Geometries," Computers \& Fluids, 39(10), 2078-2089.

Votta, R., Schettino, A., and Bonfiglioli, A., 2013, "Hypersonic High Altitude Aerothermodynamics of a Space Re-entry Vehicle," Aerospace Science and Technology, 25(1), 253-265.

Walpot, L.M., Wright, M.J., Noeding, P., and Schrijer, F., 2012, "Base Flow Investigation of the Apollo AS-202 Command Module," Progress in Aerospace Sciences, 48, 57-74.

http://dx.doi.org/10.1016/j.paerosci.2011.06.006. 